\title{
A group theoretical model of symmetry cognition
}

\author{
Jiro Hamada ${ }^{\mathrm{a}, *}$, Kaname Amano ${ }^{\mathrm{b}}$, Steve T. Fukuda ${ }^{\mathrm{c}}$, Chigusa Uchiumi ${ }^{\mathrm{d}}$, \\ Kohji Fukushi ${ }^{\mathrm{e}}$, Peter A. van der $\operatorname{Helm}^{\mathrm{f}}$ \\ a Tokushima University, Hachiman-cho, Babayama, Tokushima 770-8070, Japan \\ b Ehime University, Yunoyama, Matsuyama, Ehime 791-0121, Japan \\ c Faculty of Education, Bunkyo University, Koshigaya, Saitama 343-8511, Japan \\ d Integrated Arts and Sciences, Tokushima University, Tokushima 770-8502, Japan \\ e Faculty of Education, Kawamura Gakuen Women's University, Abiko, Chiba 270-1138, Japan \\ ${ }^{\mathrm{f}}$ Laboratory of Experimental Psychology, University of Leuven (K.U. Leuven), Tiensestraat 102, Box 3711, B-3000 Leuven, Belgium
}

\section{A R T I C L E I N F O}

\section{Article history:}

Received 12 April 2016

Received in revised form 22 August 2016

Accepted 5 October 2016

Available online $\mathrm{xxxx}$

\section{Keywords:}

Symmetry cognition

Pattern goodness and complexity

Cyclic and dihedral groups

Group theoretical model

\begin{abstract}
A B S T R A C T
We report on two experiments, published originally in Japanese, on judged goodness and simplicity of dot patterns with reflectional and rotational symmetries (with 1-4 reflection axes and repeats, respectively) under free-viewing tasks. We found that (a) both goodness and simplicity increase monotonously with the number of transformations under which a pattern is invariant; (b) stimulus outlines, such as squares and hexagons, affect both goodness and simplicity; and (c) factors such as contrast polarity and collinearity affect simplicity rather than goodness. The employed free-viewing tasks contrast with detection tasks involving short presentation times, and based on behavioural and neurophysiological evidence, we conclude that this transformational approach captures late rather than early aspects of visio-cognitive processing of visual regularities.
\end{abstract}

(c) 2016 Elsevier B.V. All rights reserved.

\section{Introduction}

Mirror symmetry is abundantly present in nature and decorative art, and it is considered to be a visual regularity, that is, a regularity to which the human visual system is sensitive. This sensitivity implies that visual regularities influence how we perceive and appreciate things. In this study, we discuss this in more detail. We focus on effects of visual regularities on judgments of stimulus complexity and figural goodness, which, in research on visual shape perception, have been seen as different notions as well as being associated with each other.

Complexity, on the one hand, usually refers to the effort - in terms of descriptive parameters - to specify a stimulus (e.g., Attneave, 1954, 1955; Chipman, 1977; Leeuwenberg, 1969, 1971). This implies, for instance, that an irregular quadrangle is more complex than an irregular triangle, because it consists of more line segments and angles. A regular quadrangle like a square, however, is simpler than an irregular triangle, because it is mirror-symmetrical and, therefore, consists of fewer different line segments and angles. The latter shows that regularities like mirror symmetry are factors that essentially reduce complexity. This contrasts with, for instance, Marković and Gvozdenović (2001) who for unclear reasons - took symmetry and simplicity as separate stimulus dimensions. However, it agrees with ideas of complexity reducing regularities being crucial in aesthetics (Birkhoff, 1933; Boselie \& Leeuwenberg, 1985; Eysenck, 1941), and more general, in perceptual

\footnotetext{
* Corresponding author.

E-mail address: jhamada@me.pikara.ne.jp (J. Hamada).
}

organization (e.g., Hochberg \& McAlister, 1953; Leeuwenberg \& van der Helm, 2013; van der Helm, 2014).

Goodness, on the other hand, is an intuitive notion that, in the past, got connotations such as learnability and rememberability of all sorts of stimuli. This was taken to be related to complexity, under the motto: A simpler stimulus is better in that it can be learned faster and remembered more accurately. Later, goodness received the connotation of detectability of regularities such as mirror symmetry, Glass patterns (i.e., randomly positioned but coherently oriented dot dipoles; Glass, 1969; Glass \& Pérez, 1973) and repetition - usually under short presentation times of less than 500 ms (e.g., Bertamini, 2010; Bruce \& Morgan, 1975; Maloney, Mitchison, \& Barlow, 1987; Wagemans, 1995; Wenderoth, 1995). A regularity then is said to be better if it is detected faster and more accurately, and if its detectability is more robust to perturbations. This detectability is known to correlate poorly with stimulus complexity. For instance, fairly independent of complexity, mirror symmetries and Glass patterns are about equally well detectable and better detectable than repetitions (van der Helm, 2010; van der Helm \& Leeuwenberg, 1996).

Here, in two experiments reported originally in Japanese, we used free-viewing tasks to investigate judged goodness and complexity of mirror symmetries and full-circle repetitions in polar coordinates, also known as reflectional and rotational symmetries, with 1-4 reflection axes and repeats, respectively. Free-viewing tasks, by which participants have ample time to make their judgments, contrast with detection tasks involving short stimulus presentation times. Whereas the latter could be said to probe early symmetry perception, the former 
could be said to probe later symmetry cognition (we return to this issue in the General discussion section). Furthermore, reflectional and rotational symmetries are so named because these patterns are invariant under transformations such as reflection and rotation. This transformational characterization has been applied, for instance, in crystallography to classify crystals by the number and type of such invariance rotations (see, e.g., Shubnikov \& Koptsik, 1974) and in research on decorative art to classify repetitive 2D motifs in wallpaper, bands, and friezes (see, e.g., Weyl, 1952).

The transformational characterization of regularities - though not the only one available (see General discussion section) - is employed in this study, and to specify it further, we consider Japanese crests (see Fig. 1). Such crests number up to a few thousands. They reflect, among other things, historical and legal embodiments associated with families, and as a rule, they contain transformational symmetries. Based on the latter, Hamada, Uchiumi, Fukushi, and Amano (2011) classified them into 8 configurations having $1-4$ invariance rotations $\left(360^{\circ}, 180^{\circ}\right.$, $120^{\circ}$, and $90^{\circ}$ ) around the center of the pattern and 1-4 reflection axes passing through the center of the pattern. In group theory, two-dimensional (2D) patterns with only invariance rotations over $360^{\circ} / n$ $(n=1,2, \ldots)$ form cyclic groups $C_{n}$, while $2 \mathrm{D}$ patterns with $n$ reflection axes $(n=1,2, \ldots)$ and invariance rotations over $360^{\circ} / n$ form dihedral groups $D_{n}$. Furthermore, a pattern generating the cyclic group $C_{n}$ has order $n$ and a pattern generating the dihedral group $\mathrm{D}_{n}$ has order $2 n$. This group order reflects the number of transformations under which a pattern is invariant. For example, in Fig. 1, the Crane $\left(C_{1}\right)$ has order 1 (1 invariance rotation over $360^{\circ}$ ); the Four Diabolos $\left(C_{4}\right)$ has order 4 (4 invariance rotations over $\left.90^{\circ}\right)$; the Hermitage $\left(D_{1}\right)$ has order 2 (1 invariance rotation over $360^{\circ}$ and 1 reflection axis); and the Four Squares $\left(D_{4}\right)$ has order 8 ( 4 invariance rotations over $90^{\circ}$ and 4 reflection axes).

In cognitive psychology, various versions of the transformational characterization of regularities have been applied to investigate figural goodness. For instance, according to Garner and Clement (1963) and Garner $(1966,1970)$, the figural goodness of a pattern does not depend on characteristics of the individual pattern but depends on the size of a pattern's rotation and reflection subset, also called the equivalence subset size (ESS). ESS specifies the number of different patterns obtained when a pattern is rotated (in steps of $90^{\circ}$ ) or reflected (horizontally, diagonally, or vertically). Under the motto "Good patterns have few alternatives", Garner postulated that pattern goodness is correlated inversely with ESS. In a similar vein, Palmer $(1982,1983)$ considered invariance under the group of Euclidean similarity transformations (rotation, translation, reflection, and dilatation) to account for figural goodness, under the motto: Invariance under more transformations implies higher goodness. Using the same motto, Imai, Ito, and Ito (1976a,b) and Imai $(1986,1992)$ considered invariance under what they called cognitive transformations (reflection, $180^{\circ}$ rotation, or both), which accounted for differences in goodness between patterns with the same ESS.

Following up on this, the present study focuses on judged goodness and complexity of cyclic $C_{n}$ patterns (i.e., rotational symmetries with group order $n$ ) and dihedral $\mathrm{D}_{n}$ patterns (i.e., reflectional symmetries with group order $2 n$ ). In Experiment 1, we consider 21-dot $\mathrm{C}_{n}$ and $\mathrm{D}_{n}$ patterns $(n=1,2,4)$ in a $9 \times 9$ matrix framework, and in Experiment 2, 19-dot $C_{n}$ and $D_{n}$ patterns $(n=1,2,3)$ in a 19cell hexagonal framework (see Fig. 2). Notice that framework size and type codetermine which values of $n$ can be investigated, and that we anticipate an effect of framework type on judged goodness and complexity.

\section{General method}

In both experiments reviewed here, stimulus patterns consisted of open and solid dots printed on white cards, with pattern numbers printed at the bottom of the card. Participants were undergraduate students from Tokushima University (they received course credits). Each participant sat in front of a desk in a normally lit room at a viewing distance of about $30 \mathrm{~cm}$. Participants were given a deck of cards, were asked to shuffle it and to rate the patterns on either goodness or complexity.

Goodness and complexity ratings were performed separately. Participants were not informed about our criteria for goodness and complexity, and were simply asked to rate goodness and complexity on 9-point scales from Poor (1) to Good (9) and from Simple (1) to Complex (9). They viewed the cards freely, which made it hard to fixate pattern orientation. Therefore, pattern orientation was treated as a random factor that was not analyzed further.

Before rating goodness or complexity, participants looked through the deck of cards once in order to grasp the outline of the patterns. Then, working at their own pace, they rated the set of cards by writing the pattern number and ratings on a response sheet. They repeated the shuffle-and-rate process twice. The first session was considered practice and only data from the second session were used for data analysis.

\section{Experiment 1}

Hamada (1988, Experiment 2) probed 13-dot compound patterns in a $7 \times 7$ matrix framework, made by superimposing 5 -dot patterns in $3 \times 3$ matrices centered on 8 -dot patterns in $4 \times 4$ matrices (without overlapping dots). The dots in these constituent matrices were all open, or all solid, or open in one matrix and solid in the other (open/ solid). He found no significant differences between the goodness ratings of open, open/solid, and solid dot patterns, while the complexity ratings of solid dot patterns were higher than those of open/solid dot patterns, which, in turn, were higher than those of open dot patterns.

Expanding on Hamada's (1988) 13-dot compound patterns, Hamada, Uchiumi, Fukushi, and Amano (2013) probed 21-dot compound patterns in a $9 \times 9$ matrix framework, composed similarly of 8dot prototype figures in $4 \times 4$ matrices and 13-dot prototype figures in $5 \times 5$ matrices (see Fig. 2a and b). For instance, in Fig. 2, the superimposition of a solid 8-dot prototype and an open 13-dot prototype in the $4 \times 4$ and $5 \times 5$ matrixes resulted in the 21 -dot compound pattern. Next, we review this experiment, which tested these compound patterns in a within-participant design to investigate effects of symmetry group order on pattern goodness and complexity.

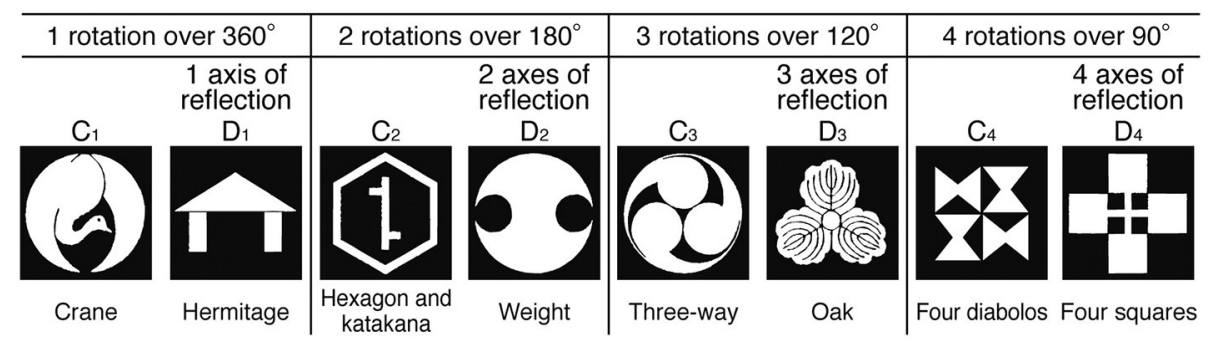

Fig. 1. Japanese crests and their symmetry groups. 
(a)

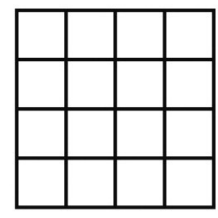

(b)

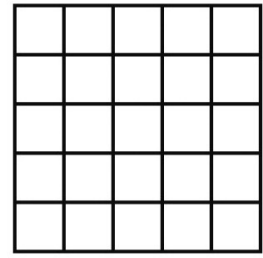

Matrix framework

for 8- and 13-dot prototype figures

(c)

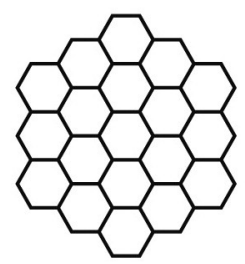

19-cell hexagonal framework

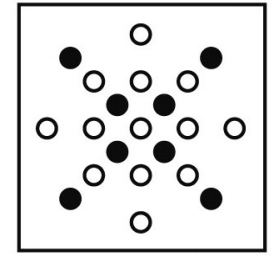

\section{1-dot compound pattern}

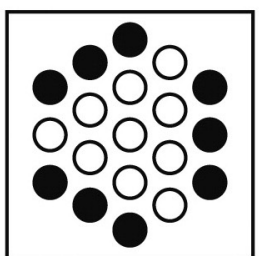

Filled pattern consisting of open and solid dots

Fig. 2. (a) and (b) Imaginary frameworks used in Experiment 1. (c) Imaginary framework used in Experiment 2. For each experiment, an example pattern is shown.

\subsection{Method}

\subsubsection{Participants}

The participants in this experiment were 208 undergraduates.

\subsubsection{Stimuli}

The 8-and 13-dot prototype figures were made using open and solid dots with a diameter of $4 \mathrm{~mm}$. The prototype figures were placed in $4 \times 4$ and $5 \times 5$ matrices, respectively, with a distance of $10 \mathrm{~mm}$ between the centers of the dots (see Fig. 2). Examples of the 21-dot compound patterns used in the experiment are shown in Fig. 3. When the 8dot and 13-dot prototype figures consisted of only open dots or only solid dots, the conditions were called homogeneous. When the 13-dot prototype figures consisted of open dots and the 8-dot prototype figures of solid dots, the condition was called open/solid. When the 13-dot prototype figures consisted of solid dots and the 8-dot prototype figures of open dots, the condition was called solid/open. The open/solid and solid/open conditions were called heterogeneous. Apart from the contrast polarity of the dots, the same 36 pattern configurations were used in all conditions. The quadrants in Fig. 3 show these 36 pattern configurations by way of 9 patterns from each of the four conditions. The patterns were printed on $70 \mathrm{~mm} \times 66 \mathrm{~mm}$ cards.

\subsubsection{Procedure}

The 208 participants in this experiment were divided into two sets of 104 participants each, one set for goodness ratings and the other set for complexity ratings. Each set of 104 participants was divided into two groups (groups A and B) of 52 participants each. Group A was exposed to the homogeneous open dots condition and heterogeneous open/ solid dots condition (see the top quadrants in Fig. 3). Group B was exposed to the homogeneous solid dots condition and heterogeneous solid/open-dots condition (see the bottom quadrants in Fig. 3). Each participant was given a deck of 72 cards to rate goodness or complexity of 36 homogeneous and 36 heterogeneous patterns. To control for the effect of orientation, each group was divided into 4 subgroups of 13 participants each, one subgroup for each of four orientations of the patterns $\left(0^{\circ}, 90^{\circ}, 180^{\circ}, 270^{\circ}\right)$.

\subsection{Results}

The Pearson's correlation coefficients between groups A and B for the 72 compound patterns, pooled over the four orientations, were high for both goodness $(r=.943, p<.001)$ and complexity $(r=.980$, $p<.001$ ). The groups were therefore pooled, and Fig. 3 shows the means of the goodness and complexity ratings for the 36 pattern configurations. The Pearson's correlation coefficient between the goodness and complexity ratings of the 72 compound patterns was high $(r=-.915, p<.001)$.

\subsubsection{Effects of contrast polarity}

Fig. 3 indicates, for each pattern configuration, the dihedral $\left(D_{1}, D_{2}\right.$, or $\left.D_{4}\right)$ or cyclic $\left(C_{1}, C_{2}\right.$, or $\left.C_{4}\right)$ group to which it belongs, and Fig. 4 shows rated goodness and complexity for the homogeneous and heterogeneous conditions in function of the order of the symmetry groups. Mean was calculated from 104 participants for the fifteen $C_{1}$, nine $C_{2}$, and three $C_{4}$ compound patterns. $t$-Tests for $C_{n}$ compound patterns yielded significant differences between the homogeneous and heterogeneous conditions for both goodness $(p<.001)$ and complexity $(p<.001)$. Similarly, means for the five $\mathrm{D}_{1}$, three $\mathrm{D}_{2}$, and one $\mathrm{D}_{4}$ compound patterns are shown in Fig. 4. $t$-Tests of homogeneous versus heterogeneous conditions for $\mathrm{D}_{n}$ compound patterns did not yield a significant difference for goodness ratings $(p=.214)$, but did yield a significant difference for complexity ratings $(p<.001)$.

\subsubsection{Effects of symmetry group order}

In function of the order of the symmetry groups, the goodness ratings of $C_{n}$ and $D_{n}$ patterns $(n=1,2,4)$ increased monotonously, while the complexity ratings decreased monotonously (see Fig. 4). This holds for both the homogeneous condition and the heterogeneous condition, which showed differences in absolute ratings (see above) but yet showed very similar tendencies in function of group order. In fact, 


\section{1-dot compound patterns}

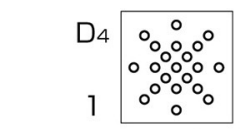

$\mathrm{Ho}: \mathrm{G}=8.1, \mathrm{C}=1.7$

$\mathrm{He}: \mathrm{G}=8.1, \mathrm{C}=2.3$

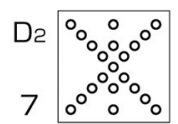

$\mathrm{Ho}: \mathrm{G}=7.5, \mathrm{C}=1.7$

$\mathrm{He}: \mathrm{G}=7.7, \mathrm{C}=2.3$

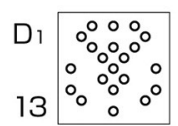

$\mathrm{Ho}: \mathrm{G}=5.9, \mathrm{C}=4.0$

$\mathrm{He}: G=5.9, C=4.7$

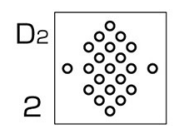

$\mathrm{G}=7.5, \mathrm{C}=2.4$

$\mathrm{G}=7.6, \mathrm{C}=2.8$

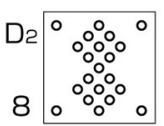

$\mathrm{G}=7.3, \mathrm{C}=2.5$

$\mathrm{G}=7.2, \mathrm{C}=3.3$

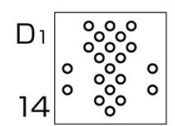

$G=5.8, C=3.7$

$\mathrm{G}=6.0, \mathrm{C}=4.3$

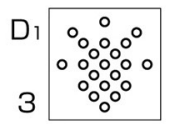

$\mathrm{G}=6.4, \mathrm{C}=3.2$

$\mathrm{G}=6.5, \mathrm{C}=3.8$

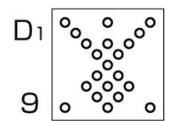

$\mathrm{G}=6.2, \mathrm{C}=3.2$

$\mathrm{G}=6.3, \mathrm{C}=3.7$

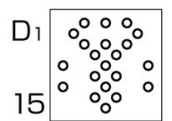

$\mathrm{G}=5.7, \mathrm{C}=3.8$

$\mathrm{G}=5.9, \mathrm{C}=4.4$

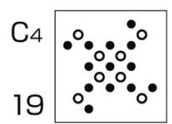

$\mathrm{Ho}: \mathrm{G}=7.1, \mathrm{C}=3.1$ $\mathrm{He}: \mathrm{G}=6.9, \mathrm{C}=4.0$

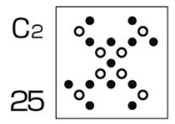

$\mathrm{Ho}: \mathrm{G}=6.1, \mathrm{C}=3.2$ $\mathrm{He}: \mathrm{G}=6.2, \mathrm{C}=4.3$

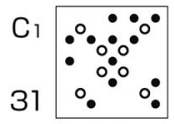

$\mathrm{Ho}: \mathrm{G}=2.5, \mathrm{C}=6.7$ $\mathrm{He}: \mathrm{G}=2.9, \mathrm{C}=7.2$

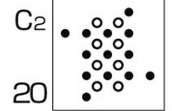

$G=5.4, C=3.8$

$\mathrm{G}=6.1, \mathrm{C}=4.2$

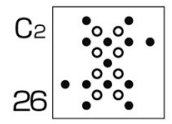

$\mathrm{G}=4.8, \mathrm{C}=4.6$

$\mathrm{G}=5.4, \mathrm{C}=4.9$

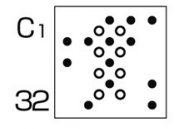

$\mathrm{G}=2.9, \mathrm{C}=6.5$

$\mathrm{G}=3.2, \mathrm{C}=6.8$

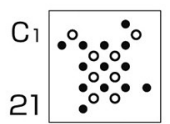

$\mathrm{G}=3.5, \mathrm{C}=5.3$ $\mathrm{G}=4.2, \mathrm{C}=5.9$
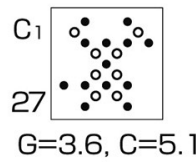

$\mathrm{G}=3.7, \mathrm{C}=6.1$

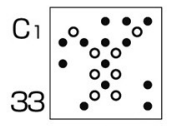

$\mathrm{G}=2.7, \mathrm{C}=6.4$ $\mathrm{G}=3.2, \mathrm{C}=7.0$

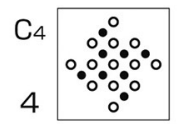

$\mathrm{G}=7.5, \mathrm{C}=2.9$

$\mathrm{G}=7.4, \mathrm{C}=3.5$

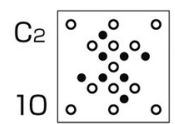

$G=5.5, C=5.0$
$G=5.9, C=5.1$

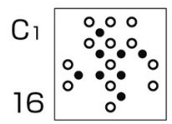

$\mathrm{G}=3.1, \mathrm{C}=6.2$ $\mathrm{G}=3.9, \mathrm{C}=6.7$

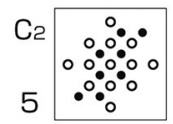

$\mathrm{G}=5.4, \mathrm{C}=4.2$

$\mathrm{G}=5.8, \mathrm{C}=4.8$

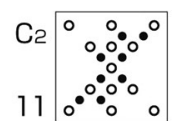

$\mathrm{G}=5.4, \mathrm{C}=3.7$

$\mathrm{G}=5.5, \mathrm{C}=4.7$

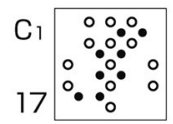

$\mathrm{G}=3.0, \mathrm{C}=6.3$

$\mathrm{G}=3.6, \mathrm{C}=6.6$

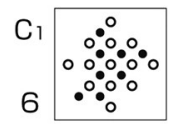

$\mathrm{G}=4.1, \mathrm{C}=4.8$

$\mathrm{G}=4.4, \mathrm{C}=5.4$

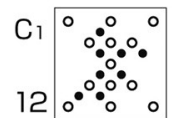

$\mathrm{G}=3.8, \mathrm{C}=5.8$

$\mathrm{G}=4.1, \mathrm{C}=5.8$

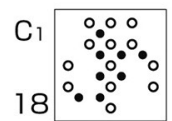

$G=3.1, C=6.2$ $G=3.3, C=6.7$

\section{Ho : Homogeneous condition \\ G : Goodness \\ He : Heterogeneus condition \\ C : Complexity}

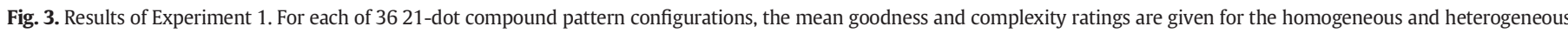
conditions.

these tendencies were so similar that the Pearson's correlation coefficients between the two conditions were $r=.999(p<.001)$ for both goodness and complexity. Therefore, the two conditions were pooled for further analysis.

$t$-Tests of rated goodness yielded significant differences $(p<.001)$ between $\mathrm{D}_{n}$ and $\mathrm{C}_{2 n}$ patterns $(n=1,2)$. That is, $\mathrm{D}_{1}$ compound patterns (mean $M=6.1$ ) were better than $C_{2}$ compound patterns $(M=5.6)$, and $\mathrm{D}_{2}$ compound patterns $(M=7.5)$ were better than $\mathrm{C}_{4}$ compound patterns $(M=6.8)$. Hence, the goodness ratings of $\mathrm{D}_{n}$ patterns are higher than those of $C_{2 n}$ patterns $(n=1,2)$, even though they have the same group order. $t$-Tests of rated complexity also yielded significant differences $(p<.001)$ between $D_{n}$ and $C_{2 n}$ patterns $(n=1,2)$. That is, $C_{2}$ compound patterns $(M=4.5)$ were more complex than $\mathrm{D}_{1}$ compound patterns $(M=3.9)$, and $C_{4}$ compound patterns $(M=3.8)$ were more complex than $\mathrm{D}_{2}$ compound patterns $(M=2.5)$. Hence, the complexity ratings of $D_{n}$ patterns are lower than those of $C_{2 n}$ patterns $(n=1,2)$, even though they have the same group order. In other words, goodness and simplicity (the inverse of complexity) of both $C_{n}$ and $D_{n}$ patterns increased monotonously with the order of the symmetry groups, but with group order having higher weights for $D_{n}$ than for $C_{n}$ (i.e., for each group order, $D_{n}$ patterns yielded higher goodness and simplicity values than $C_{n}$ patterns).

\subsection{Discussion}

For the 21-dot compound patterns in the $9 \times 9$ matrix framework, goodness ratings for both $C_{n}$ and $D_{n}$ compound patterns $(n=1,2,4)$ increased monotonously with the order of the symmetry groups, but with a higher group order weight for $D_{n}$ than for $C_{n}$ (see Fig. 4). This weight difference is not consistent with the transformational approaches mentioned in the Introduction section, which related goodness to symmetry group order without differentiating between $C_{n}$ and $D_{n}$ patterns. However, it is consistent with Palmer's (1991) finding that, for 9- or 10-dot patterns in a $5 \times 5$ matrix, the goodness of $D_{n}$ and $C_{n}$ patterns $(n=1$, 2,4 ) increased monotonously with the order of the symmetry groups, but with a higher group order weight for $D_{n}$ than for $C_{n}$. This suggests that, irrespective of the number of dots or the size of the matrix, 


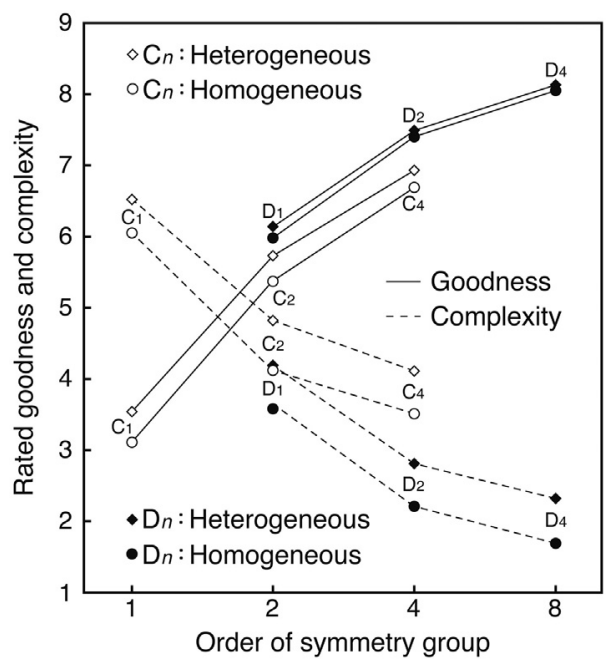

Fig. 4. Rated goodness and complexity in Experiment 1 as function of the order of symmetry groups.

goodness depends on symmetry group order but with differences between types of symmetry groups.

Furthermore, we found that the goodness ratings of the $C_{n}$ compound patterns and the complexity ratings of both $C_{n}$ and $D_{n}$ patterns under the heterogeneous conditions were higher than those under the homogeneous conditions. For the goodness ratings of $\mathrm{D}_{n}$ compound patterns, however, we found no significant difference between the homogeneous and heterogeneous conditions. This exception suggests that goodness and complexity of $\mathrm{D}_{n}$ compound patterns might be processed differently in the cognitive system. It also agrees with Wenderoth (1996) who, using dot patterns on a grey background, investigated the effects of contrast polarity on the detection of bilateral symmetry. He found the same results for (a) all dots being either black or white and (b) half of the dots being black and the other half being white (with positive correspondence between symmetrical pairs of dot). This suggests, as concluded by Tyler and Hardage (1996), that detection of mirror symmetry is mediated predominantly by polarity-insensitive mechanisms. Future studies may investigate if such mechanisms are consistent with the above-mentioned differences, which we nevertheless did find.

\section{Experiment 2}

Hamada (1988, Experiment 1) designed a 19-cell hexagonal framework (Fig. $2 \mathrm{c}$ ) with $\mathrm{C}_{n}$ and $\mathrm{D}_{n}$ filled patterns $(n=1,2,3)$ consisting of 19 open and solid dots. He investigated the effects of the order of symmetry groups as well as the effects of collinear and non-collinear elements on goodness and complexity. His results can be summarized as follows: (a) Goodness of the filled patterns increased with the order of the symmetry groups, but, this time, with a higher group order weight for $C_{n}$ than for $\mathrm{D}_{n}$ ( see also Hamada \& Ishihara, 1988), and (b) complexity of collinear element patterns decreased with the order of cyclic and dihedral groups without a weight difference between $C_{n}$ and $D_{n}$, whereas that of non-collinear element patterns was medium, regardless of group order. This contrasts with our results for the matrix framework in Experiment 1 , where we found that the goodness of $D_{n}$ patterns was higher than that of $C_{2 n}$ patterns. To further investigate this goodness reversal for the two frameworks, Hamada et al. (2013, Experiment 2) conducted the experiment that is reviewed here. It probed two additional aspects.

First, for patterns in a matrix framework, Garner and Clement (1963) and Matsuda (1978) found an effect of collinearity on goodness, and for patterns in a hexagonal framework, Hamada (1988) found an effect of collinearity on complexity but not on goodness. This was investigated further in the experiment reviewed here.

Second, Hamada (1988) used filled patterns with 9 solid and 10 open dots as well as their contrast-reversed patterns with 9 open and 10 solid dots. In both cases, the solid-dot configuration tends to be perceived as figure against an open-dot background, so that the contrast reversal also implies a figure-ground reversal. Hamada (1988) did not analyze the effect of this difference in contrast polarity on goodness and complexity, but in the experiment reviewed here, this analysis was included.

\subsection{Method}

\subsubsection{Participants}

The participants in this experiment were 144 undergraduates.

\subsubsection{Stimuli}

The stimuli consisted of 17 filled patterns with 9 solid and 10 open dots (S-patterns; see Fig. 5) and 17 filled patterns with 9 open and 10 solid dots (O-patterns). The S-patterns consisted of (a) collinear element patterns composed of ten open dots and three identical sets of three collinear non-overlapping solid dots, and (b) non-collinear element patterns composed of ten open dots and nine solid dots that did not form three identical sets of three collinear non-overlapping solid dots. One collinear element pattern was designed for each of the $D_{3}$, $D_{2}, D_{1}, C_{2}$, and $C_{1}$ groups (a collinear $C_{3}$ pattern is not possible in this framework). Additionally, two non-collinear element patterns were designed for each of the six symmetry groups. The O-patterns were created by reversing the contrast polarities of the dots in the S-patterns.

Furthermore, S- and O-patterns were rotated by $60^{\circ}, 120^{\circ}, 180^{\circ}$, $240^{\circ}$, and $300^{\circ}$ to make rotated filled patterns. Thus, the total number of filled patterns used was 204. The filled patterns were printed on white cards of $65 \mathrm{~mm} \times 62 \mathrm{~mm}$. The diameter of each dot was $7 \mathrm{~mm}$ and the distance between the centers of adjacent dots was $10 \mathrm{~mm}$.

\subsubsection{Procedure}

The 144 participants in this experiment were divided into two sets of 72 participants each, one set for goodness ratings and the other set for complexity ratings. To randomize the $\mathrm{S}$ - and $\mathrm{O}$-patterns and pattern orientations, each set was divided into groups $\mathrm{A}, \mathrm{B}$, and $\mathrm{C}$, which contained 24 participants each. Each participant judged goodness or complexity of 68 patterns (17 S-patterns plus 17 O-patterns, multiplied by 2 orientations).

\subsection{Results}

The Pearson's correlation coefficients for goodness were high between groups A and B $(r=.801, p<.001)$, groups B and C $(r=.888$, $p<.001)$, and groups $A$ and $C(r=.899, p<.001)$, as well as for complexity between groups A and B $(r=.880, p<.001)$, groups B and $\mathrm{C}(r=.920, p<.001)$, and groups $\mathrm{A}$ and $\mathrm{C}(r=.933, p<.001)$. Fig. 5 shows the means of rated goodness and complexity, pooled over the six pattern orientations. Table 1 shows means of rated goodness and complexity in function of the order of the symmetry groups and $t$-test results.

\subsubsection{Effects of symmetry group order}

Table 1 shows that, for $\mathrm{S}$ - and $\mathrm{O}$-patterns as well as for collinear and non-collinear element patterns, goodness ratings increased with the order of the cyclic and the dihedral groups, but this time, with a higher group order weight for $C_{n}$ than for $D_{n}$ (Fig. 6 shows this for the pooled data). That is, the means of the goodness ratings differed significantly $(p<.001)$ between the $C_{2}$ patterns $(M=5.2)$ and the $D_{1}$ patterns $(M=4.5)$, even though they both have symmetry group order 2 .

Table 1 and Fig. 6 show further that, for the means of pooled data, the complexity ratings of the $C_{3}$ patterns were higher than those of the $C_{2}$ 

$\mathrm{D}_{3}$
$\mathrm{C}_{3}$
$\mathrm{D}_{2}$
$\mathrm{C}_{2}$
$D_{1}$
$\mathrm{C}_{1}$

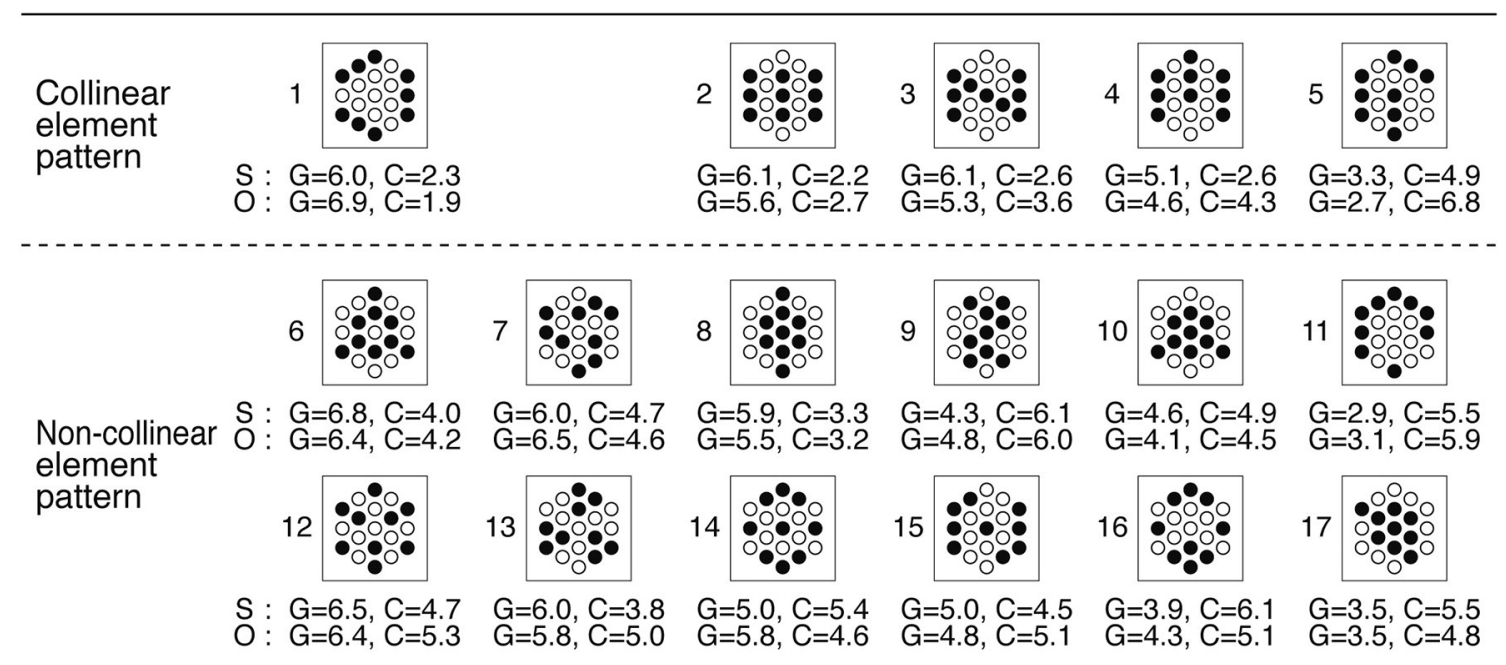

\section{$S: 9$ solid-dot filled pattern \\ G: Goodness \\ O: 9 open-dot filled pattern \\ C: Complexity}

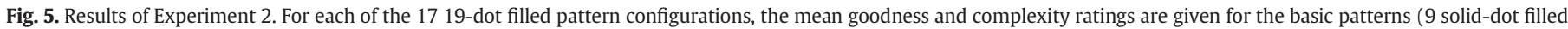
patterns) and for their open-solid reversals ( 9 open-dot filled patterns).

patterns. This is probably an outlier due to the fact that the $C_{3}$ patterns were all non-collinear patterns (as said, collinear $C_{3}$ patterns were not possible). For the rest, the complexity ratings decreased with the order of the cyclic and the dihedral groups, without a difference in group order weight. Here, there was no significant difference between $\mathrm{C}_{2}$ and $\mathrm{D}_{1}$ patterns in the means of the complexity ratings (both $M=$ 4.3).

\subsubsection{Effects of contrast polarity}

As shown in Table 1 , the mean of the goodness ratings of S-patterns was not significantly higher than that of O-patterns $[M(S)=5.2$, $M(0)=5.1, p=.101]$. There were also no separate significant differences for the $C_{n}$ patterns $[M(S)=4.9, M(0)=4.7, p=.056]$ and the $\mathrm{D}_{n}$ patterns $[M(S)=5.6, M(0)=5.5, p=.641]$.
The mean of the complexity ratings of S-patterns was significantly lower than that of O-patterns $[M(S)=4.0, M(0)=4.4, p<.001]$. Here, this difference was due to both the $C_{n}$ patterns $[M(S)=4.5$, $M(0)=5.2, p<.001]$ and the $\mathrm{D}_{n}$ patterns $[M(S)=3.5, M(0)=3.7$, $p<.01]$.

\subsubsection{Effects of collinearity}

As shown in Table 1, there was no significant difference in goodness ratings between collinear $(C)$ and non-collinear $(N C)$ element patterns $[M(C)=5.2, M(N C)=5.1, p=.379]$. There was also no such difference for the $\mathrm{D}_{n}$ patterns $[M(C)=5.7, M(N C)=5.4, p=.112]$, but there was such a difference for the $C_{n}$ patterns $[M(C)=4.4, M(N C)=4.7$, $p<.001]$.

Regarding complexity rating, there was a significant difference between collinear and non-collinear element patterns $[M(C)=3.4$,

Table 1

Means of goodness and complexity ratings in Experiment 2.

\begin{tabular}{|c|c|c|c|c|c|c|c|}
\hline & $\mathrm{C}_{1}$ & $\mathrm{C}_{2}$ & $\mathrm{C}_{3}$ & $\mathrm{D}_{1}$ & $\mathrm{D}_{2}$ & $\mathrm{D}_{3}$ & Mean \\
\hline \multicolumn{8}{|l|}{ Goodness } \\
\hline S-pattern & 3.3 & 5.4 & 6.0 & 4.6 & 5.8 & 6.3 & 5.2 \\
\hline O-pattern & 3.0 & 5.0 & 6.2 & 4.4 & 5.6 & 6.6 & 5.1 \\
\hline$t$-Test & $p<.05$ & $p<.001$ & ns & $p<.05$ & ns & $p<.01$ & ns \\
\hline Collinear & 3.0 & 5.7 & & 4.8 & 5.9 & 6.4 & 5.2 \\
\hline Non-collinear & 3.2 & 4.8 & 6.1 & 4.2 & 5.5 & 6.5 & 5.1 \\
\hline$t$-Test & $p<.05$ & $p<.001$ & & $p<.001$ & ns & ns & ns \\
\hline $\begin{array}{l}\text { Mean of } \\
\text { pooled data }\end{array}$ & 3.1 & 5.2 & 6.1 & 4.5 & 5.7 & 6.5 & 5.1 \\
\hline \multicolumn{8}{|l|}{ Complexity } \\
\hline S-pattern & 5.2 & 3.9 & 4.3 & 4.1 & 3.3 & 3.3 & 4.0 \\
\hline O-pattern & 6.1 & 4.6 & 4.8 & 4.6 & 3.3 & 3.3 & 4.4 \\
\hline$t$-Test & $p<.001$ & $p<.001$ & $p<.001$ & $p<.001$ & ns & ns & $p<.001$ \\
\hline Collinear & 5.9 & 3.1 & & 3.5 & 2.4 & 2.1 & 3.4 \\
\hline Non-collinear & 5.4 & 5.4 & 4.5 & 5.1 & 4.1 & 4.5 & 4.9 \\
\hline$t$-Test & $p<.001$ & $p<.001$ & & $p<.001$ & $p<.001$ & $p<.001$ & $p<.001$ \\
\hline $\begin{array}{l}\text { Mean of } \\
\text { pooled data }\end{array}$ & 5.6 & 4.3 & 4.5 & 4.3 & 3.3 & 3.3 & 4.2 \\
\hline
\end{tabular}




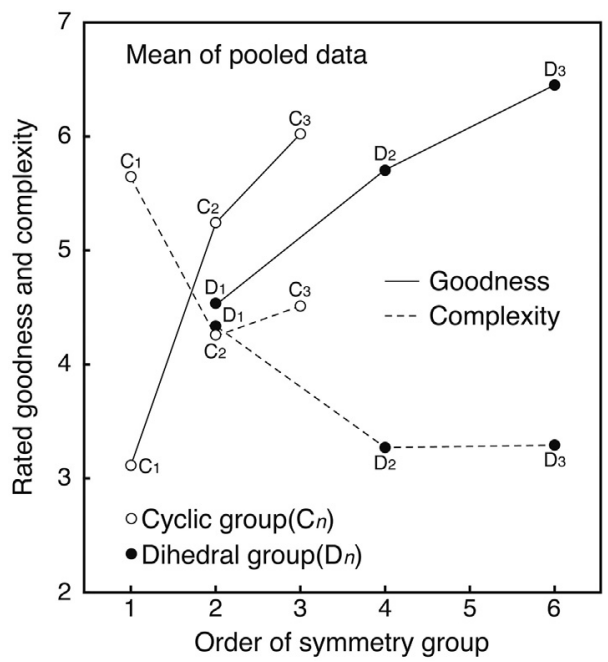

Fig. 6. Rated goodness and complexity in Experiment 2 as function of the order of symmetry groups.

$M(N C)=4.9, p<.001]$. Here, such a difference was found for both the $\mathrm{D}_{n}$ patterns $[M(C)=2.7, M(N C)=4.6, p<.001]$ and the $C_{n}$ patterns $[M(C)=4.5, M(N C)=5.1, p<.001]$.

\subsection{Discussion}

In Experiment 2, goodness and complexity of filled patterns in a hexagonal framework were measured. The results showed that the goodness and complexity ratings mirrored the results of Hamada (1988, Experiment 1), with exception to the complexity ratings of the non-collinear element patterns, which, in Hamada (1988), were medium irrespective of the order of the cyclic and dihedral groups.

\subsubsection{Goodness}

First, in Experiment 2, just as in Experiment 1, goodness ratings increased monotonously with group order, but this time, with a higher group order weight for $C_{n}$ than for $D_{n}$. The latter finding for filled patterns in a hexagonal framework is similar to that found by Hamada (1988) and Hamada and Ishihara (1988). It differs, however, from the finding for non-filled compound patterns in the matrix framework in Experiments 1, where we found a higher group order weight for $D_{n}$ than for $C_{n}$. This weight reversal seems due to the difference in framework (the difference between filled and non-filled patterns does not seem relevant; see Matsuda, 1978; Hamada \& Ishihara, 1988). Furthermore, the goodness ratings for $\mathrm{D}_{n}$ filled patterns in the hexagonal framework in Experiment 2 were clearly lower than those for $\mathrm{D}_{n}$ compound patterns in the matrix framework in Experiments 1. Also this seems due to the difference in framework.

Second, in Experiment 2, we neither found a significant difference in goodness ratings between $\mathrm{S}$ - and O-patterns for $\mathrm{C}_{n}$ nor for $\mathrm{D}_{n}$. This is in partial agreement with Experiment 1, in which we found a significant difference in goodness ratings between the homogeneous and heterogeneous conditions for $C_{n}$ but not for $D_{n}$.

Third, whereas Garner and Clement (1963) and Matsuda (1978) found an overall effect of collinearity on goodness for patterns in a matrix framework, Hamada and Ishihara (1988) did not find such an effect for patterns in a hexagonal framework. Here, for patterns in the hexagonal framework in Experiment 2, we now found a significant effect of collinearity on goodness for $C_{n}$ but not for $\mathrm{D}_{n}$.

In sum, the type of framework seems to have a clear effect on the goodness of $\mathrm{D}_{n}$ patterns and on whether they are better or worse than
$\mathrm{C}_{n}$ patterns. Furthermore, contrast polarity and collinearity do not show consistent overall effects - if anything, they seem to affect the goodness of $C_{n}$ patterns rather than that of $D_{n}$ patterns.

\subsubsection{Complexity}

First, in Experiment 2, we found that, except for the $C_{3}$ patterns, complexity ratings for patterns in the hexagonal framework decreased with the order of symmetry groups, without a difference in group order weight between cyclic and dihedral groups (see also Hamada \& Ishihara, 1988). This contrasts with our findings for patterns in the matrix framework in Experiment 1, where complexity ratings also decreased with the order of symmetry groups, but with $\mathrm{D}_{n}$ patterns yielding lower complexity (or higher simplicity) values than $C_{2 n}$ patterns. This difference in dependency on group order seems again due to the difference in framework.

Second, in Experiment 2, we found significant differences in complexity ratings between S- and O-patterns for both $C_{n}$ and $D_{n}$. This is consistent with Experiment 1, where we found significant differences in complexity ratings between the homogeneous and heterogeneous conditions for both $C_{n}$ and $D_{n}$. This suggests that, in either type of framework, contrast polarity has an overall effect on complexity, which may be related to an implied figure-ground reversal.

Third, for patterns in the hexagonal framework in Experiment 2, we found that collinear patterns were rated significantly simpler than noncollinear patterns, for both $C_{n}$ and $D_{n}$. This agrees with Hamada's (1988) findings, which also applied to patterns in a hexagonal framework. Again, future research may show if this holds for matrix frameworks too.

In sum, the type of framework seems to have an effect on the dependency of complexity on the order of cyclic and dihedral groups. Furthermore, contrast polarity and collinearity seem to have a consistent overall effect on the complexity of both $C_{n}$ and $D_{n}$ patterns.

\section{General discussion}

In this article, we discussed two experiments on symmetry cognition. Both experiments involved free-viewing tasks probing judged goodness and judged complexity of dot patterns. Experiment 1 employed 21-dot cyclic $C_{n}$ and dihedral $D_{n}$ patterns $(n=1,2,4)$ in a $9 \times 9$ matrix framework, while Experiment 2 employed 19-dot $C_{n}$ and $\mathrm{D}_{n}$ patterns $(n=1,2,3)$ in a 19-cell hexagonal framework. In both experiments, our main objective was to investigate the effect of the transformational group order of the cyclic and dihedral patterns on goodness and complexity ratings (group order is $n$ in case of $C_{n}$ and $2 n$ in case of $\mathrm{D}_{n}$ ). Our results on this main point showed both parallels and differences between the two types of frameworks. This can be cast in a model as follows (see Fig. 7).

\subsection{A group theoretical model of symmetry cognition}

Overall, we found that goodness and simplicity (the inverse of complexity) increase monotonously with group order, but with the following differences between the two types of frameworks. Regarding goodness, the group order weight for $\mathrm{D}_{n}$ patterns is higher than that for $C_{n}$ patterns in the matrix framework, but lower in the hexagonal framework. Furthermore, regarding simplicity, the group order weight for $D_{n}$ patterns is higher than that for $C_{n}$ patterns in the matrix framework, but equal in the hexagonal framework.

For goodness, neither the weight differences between $D_{n}$ and $C_{n}$ in either framework nor the weight reversal between the two frameworks is implied by the transformational approaches mentioned in the Introduction section, which did not differentiate between $\mathrm{D}_{n}$ and $\mathrm{C}_{n}$. The weight reversal, in particular, suggests that the type of framework is a goodness factor that should be taken into account (see also next subsection). The type of framework seems relevant also for simplicity, 
J. Hamada et al. / Acta Psychologica 171 (2016) xxx-xxx

A group theoretical model of symmetry cognition

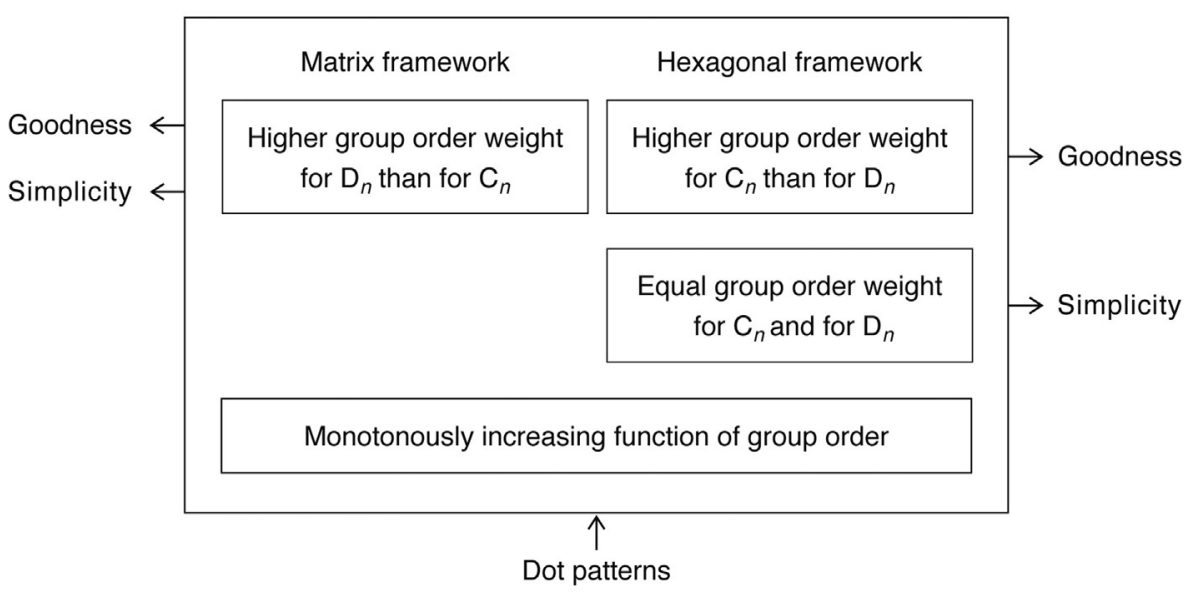

Fig. 7. A group theoretical model of symmetry cognition.

considering the weight difference between $\mathrm{D}_{n}$ and $\mathrm{C}_{n}$ in the matrix framework but not in the hexagonal framework.

Our findings for the hexagonal framework suggest further that goodness (weight difference between $\mathrm{D}_{n}$ and $\mathrm{C}_{n}$ ) and simplicity (no weight difference between $\mathrm{D}_{n}$ and $\mathrm{C}_{n}$ ) are differently processed concepts. This is corroborated by our findings for contrast polarity (in both experiments) and collinearity (in Experiment 2). That is, we found that these factors have a consistent overall effect on simplicity but not on goodness.

In sum, our findings suggest not only that goodness and simplicity are different concepts but also that, to both concepts, the transformational group order of patterns is a pivotal factor. In addition, we found that goodness and simplicity can be modulated by other factors, such as the type of framework, contrast polarity, and collinearity.

\subsection{Symmetry cognition versus symmetry perception}

As mentioned in the Introduction section, the free-viewing judgment tasks in the current experiments contrast with detection tasks involving short presentation times (under $500 \mathrm{~ms}$ ). The latter tasks could be said to probe early symmetry perception rather than later symmetry cognition. The border between perception and higher cognitive levels is admittedly fuzzy, but notice that we can detect stimulus features like mirror symmetry under presentation times as short as $50 \mathrm{~ms}$ (e.g., Locher \& Wagemans, 1993; Csathó, van der Vloed, \& van der Helm, 2003), while complete percepts are formed within $500 \mathrm{~ms}$ (e.g., Sekuler \& Palmer, 1992; Breitmeyer \& Ogmen, 2006). To discuss this issue further, we next compare the transformational ideas that guided the current experiments to the non-transformational ideas in van der Helm and Leeuwenberg's (1996) holographic approach to visual regularities, which was developed specifically to capture early symmetry perception.

Transformational ideas on visual symmetry, on the one hand, rely on invariance under motion. For instance, a mirror-symmetrical pattern with one or more reflection axes (i.e., a $\mathrm{D}_{n}$ pattern) is invariant under $180^{\circ}$ three-dimensional rotations about the reflection axes; an (infinite) translational symmetry, or a repetition, is invariant under 2D longitudinal translations over one or more repeats; and a rotational symmetry (i.e., a $C_{n}$ pattern, or a full-circle repetition in polar coordinates) is invariant under 2D rotations over one or more repeats. These invariance transformations identify pattern blocks (i.e., symmetry halves or repeats) with each other in one go. So, transformationally, mirror symmetries, translational symmetries, and rotational symmetries can all be said to have a block structure. Furthermore, a pattern is predicted to be better if it is invariant under more transformations (which, albeit with some nuances, we confirmed in this article).

The holographic approach to visual symmetry, on the other hand, relies on invariance under growth. That is, it holds that visual regularities are configurations that can be expanded (i.e., can grow) preserving the regularity in them. For instance, preserving the regularity in them, a mirror symmetry can be expanded by one symmetry pair at a time, a repetition by one repeat at a time, and a Glass pattern by one dot dipole at a time. The holographic approach can therefore be said to assign a point structure to mirror symmetry, a block structure to repetition, and a dipole structure to Glass patterns. Furthermore, the holographic model basically relies on two stages: a first stage yielding the simplest structural description of a stimulus, and a second stage in which the goodness of a regularity is determined by the weight-of-evidence for the regularity in this simplest structural description (see below). Notice that it defines the complexity of a structural description by the number of parameters needed to specify a stimulus. This may deviate from judged complexity as considered in this article, but just as we concluded here, the two stages in the holographic model suggest that simplicity and goodness are different concepts.

The holographic weight-of-evidence for a regularity in a stimulus exploits the holographic structure differences between mirror symmetries, repetitions, and Glass patterns. That is, it amounts roughly to the number of symmetry pairs, repeats, or dipoles, respectively, normalized by the total number of elements in a stimulus. This model explains a wide range of goodness phenomena found, notably, in behavioural experiments using short presentation times (for an overview, see van der Helm, 2014). For instance, it explains that single mirror symmetries (i.e., $\mathrm{D}_{1}$ patterns) and Glass patterns are about equally good and equally susceptible to perturbations (this is not implied by transformational models, which do not cover Glass patterns and which cannot deal with perturbations). Furthermore, it explains that the goodness of single mirror symmetries and Glass patterns is independent of the number of elements in a pattern (for mirror symmetries, this is also implied by transformational models). Moreover, it explains that these regularities are generally better than repetitions (not implied by transformational models), while the goodness of repetitions depends on both the number of repeats (also implied by transformational models) and the number of elements per repeat (not implied by transformational models).

Hence, the goodness predictions by the transformational and holographic approaches are different but overlap partly. Currently relevant, this also holds for the goodness of multiple mirror symmetries (i.e., $\mathrm{D}_{n}$ patterns, $n=1,2,3, \ldots$. .). Both approaches predict that goodness increases monotonously for $n=1,2,4,8$, etc. However, on the one hand, the transformational approach predicts that the goodness for 
other values of $n$ follows this monotonous increase, which could be captured by $1-1 /(2 n)$ for all $n$. On the other hand, the holographic approach predicts that the goodness for other values of $n$ deviates downward from this monotonous increase. This is due to the first stage in the holographic model. That is, for $n=1,2,4$, 8, etc., the simplest structural description of a $D_{n}$ pattern specifies it as a multiple mirror symmetry, but for other values of $n$, it specifies it as a repetition in polar coordinates (i.e., as a $C_{n}$ pattern), which yields a lower weightof-evidence (van der Helm, 2011).

In particular, transformationally, the goodness of $D_{3}$ patterns would fit in the monotonous increase captured by $1-1 /(2 n)$, whereas the holographic approach predicts that they are worse than $\mathrm{D}_{2}$ patterns. Wenderoth and Welsh (1998) pitted these predictions against each other for $\mathrm{D}_{n}(n=1,2,3,4)$. They found that the goodness of $\mathrm{D}_{3}$ patterns deviates from the monotonous increase for the other values of $n$ and that $D_{3}$ patterns are not better than $D_{2}$ patterns. Furthermore, holographically, a $\mathrm{D}_{4}$ pattern like a square is indeed a multiple mirror symmetry, but a $D_{6}$ pattern like a hexagon is a multiple rotational symmetry (i.e., a $\mathrm{C}_{6}$ pattern). This may explain that a square framework favors $D_{n}$ patterns over $C_{n}$ patterns (as found in Experiment 1 ), while a hexagonal framework favors $C_{n}$ patterns over $D_{n}$ patterns (as found in Experiment 2).

The foregoing comparison of the transformational and holographic approaches raises the question whether they are mutually exclusive or perhaps yet reconcilable. We think they are partly reconcilable, namely, by relating their predictions for multiple mirror symmetries to different time windows in visio-cognitive processing. As mentioned, the holographic approach was developed specifically to capture early symmetry perception as probed by detection tasks involving short presentation times. The transformational approach, conversely, seems appropriate to capture later symmetry cognition as probed in this article by free-viewing judgment tasks. In other words, it may be that the holographic approach captures early aspects of visio-cognitive processing, while the transformational approach captures later aspects.

This differentiation is corroborated by the electroencephalography study by Makin et al. (2016; see also Bertamini \& Makin, 2014; Makin, Rampone, Pecchinenda, \& Bertamini, 2013). They tested if the event related potential called the sustained posterior negativity (SPN) is an electrophysiological index of the perceptual goodness of visual regularities. They found that, in a time window of 300-1000 ms after stimulus onset, the SPN correlates highly with the holographic weight-of-evidence. This holds, for instance, for (a) the absence of a difference between single mirror symmetries and Glass patterns, (b) the difference between them and 2-fold translational or rotational repetitions, and (c) the dependency on the total number of pattern elements in repetitions but not in mirror symmetries (as discussed, these aspects are not implied by transformational models). Their currently most relevant finding applies to multiple mirror symmetries (i.e., $\mathrm{D}_{n}$ patterns, $n=1-5$ ). In a "perceptual" time window of 350-450 ms after stimulus onset, the SPN correlated nearly perfectly with the holographic weight-of-evidence (i.e., with $D_{3}$ and $D_{5}$ deviating downward from the monotonous increase with $n$ for the other $n$ ). In a more "cognitive" window of 600 $1000 \mathrm{~ms}$ after stimulus onset, however, the SPN correlated best with the transformationally predicted monotonous increase with $n$ for all $n$. Hence, not only the difference in behavioural tasks (detection under short presentation times versus free-viewing judgment) but also this electrophysiological evidence suggests that, regarding multiple mirror symmetries, both the holographic and transformational models might be correct, but for different time windows after stimulus onset.

\section{Conclusion}

In this article, we discussed two experiments on symmetry cognition, using free-viewing tasks. We considered $\mathrm{D}_{n}$ patterns $(\mathrm{n}=1-4$; i.e., reflectional symmetries or mirror-symmetrical patterns with 1-4 reflection axes) and $\mathrm{C}_{n}$ patterns $(\mathrm{n}=1-4$; i.e., rotational symmetries or full-circle repetitions in polar coordinates with $1-4$ repeats). We found that both the judged goodness and the judged simplicity of these patterns increase monotonously with the number of transformations under which they are invariant. We also found, however, that judged goodness and judged simplicity are differently processed concepts, which are differentially modulated by other factors, such as stimulus outline, contrast polarity, and collinearity. Finally, the employed free-viewing tasks contrast with tasks involving short presentation times, and based on both behavioural and neurophysiological evidence, we conclude that the transformational approach captures late rather than early aspects of visio-cognitive processing of visual regularities.

\section{Acknowledgments}

We are greatly indebted to Shiro Imai and Toru Ishihara who supported this research from the beginning. We are also grateful to Galina Paramei for her valuable comments on our study. The research of PvdH was supported by Methusalem grant METH/14/02 awarded to Johan Wagemans (www.gestaltrevision.be).

\section{References}

Attneave, F. (1954). Some informational aspects of visual perception. Psychological Review, 61, 183-193.

Attneave, F. (1955). Symmetry, information, and memory for patterns. American Journal of Psychology, 68, 209-222.

Bertamini, M. (2010). Sensitivity to reflection and translation is modulated by objectness. Perception, 39, 27-40.

Bertamini, M., \& Makin, A. D. J. (2014). Brain activity in response to visual symmetry. Symmetry, 6, 975-996.

Birkhoff, G. D. (1933). Aesthetic measure. Cambridge, MA: Harvard University Press.

Boselie, F., \& Leeuwenberg, E. L. J. (1985). Birkhoff revisited: Beauty as a function of effect and means. American Journal of Psychology, 98, 1-39.

Breitmeyer, B. G., \& Ogmen, H. (2006). Visual masking: time slices through conscious and unconscious vision. Oxford, UK: Oxford University Press.

Bruce, V. G., \& Morgan, M. J. (1975). Violations of symmetry and repetition in visual patterns. Perception, 4, 239-249.

Chipman, S. F. (1977). Complexity and structure in visual patterns. Journal of Experimental Psychology: General, 106, 269-301.

Csathó, Á., van der Vloed, G., \& van der Helm, P. A. (2003). Blobs strengthen repetition but weaken symmetry. Vision Research, 43, 993-1007.

Eysenck, H. J. (1941). The empirical determination of an aesthetic formula. Psychological Review, 48, 83-92.

Garner, W. R. (1966). To perceive is to know. American Psychologist, 21, 11-19.

Garner, W. R. (1970). Good patterns have few alternatives. American Scientist, 58, 34-42. Garner, W. R., \& Clement, D. E. (1963). Goodness of patterns and pattern uncertainty. Journal of Verbal Learning and Verbal Behavior, 2, 446-452.

Glass, L. (1969). Moiré effect from random dots. Nature, 223, 578-580.

Glass, L., \& Pérez, R. (1973). Perception of random dot interference patterns. Nature, 246, $360-362$.

Hamada, J. (1988). Effects of symmetry groups on complexity and goodness in visual pattern. Japanese Journal of Psychology, 59, 137-143 Japanese text with English abstract.

Hamada, J., \& Ishihara, T. (1988). Complexity and goodness of dot patterns varying in symmetry. Psychological Research, 50, 155-161.

Hamada, J., Uchiumi, C., Fukushi, K., \& Amano, K. (2011). Complexity and goodness of dot patterns depending upon symmetry groups. Japanese Psychological Review, 54, 138-152 Japanese text with English abstract.

Hamada, J., Uchiumi, C., Fukushi, K., \& Amano, K. (2013). Goodness and complexity of compound and filled patterns depending upon a hierarchy of perception. Japanese Journal of Psychonomic Science, 31, 123-134 Japanese text with English abstract and figure captions.

Hochberg, J. E., \& McAlister, E. (1953). A quantitative approach to figural "goodness". Journal of Experimental Psychology, 46, 361-364.

Imai, S. (1986). The transformational structure theory of pattern cognition. Japanese Psychological Monographs, 17, Tokyo: Tokyo University Press Japanese text with English summary.

Imai, S. (1992). Fundamentals of cognitive judgments of pattern. In G. H.G., S. W. Link, \& J. T. Townsend (Eds.), Cognition, information processing, and psychophysics: Basic issues (pp. 225-265). New Jersey: Lawrence Erlbaum Associates.

Imai, S., Ito, T., \& Ito, S. (1976a). Effect of intra-pattern transformation structure upon goodness judgments of two-dimensional patterns. Japanese Journal of Psychology, 47, 202-210 Japanese text with English abstract.

Imai, S., Ito, S., \& Ito, T. (1976b). Effects of intra-pattern transformation structures and the number of runs upon goodness and complexity judgments of patterns. Japanese Psychological Review, 19, 77-94 (Japanese text with English abstract).

Leeuwenberg, E. L. J. (1969). Quantitative specification of information in sequential patterns. Psychological Review, 76, 216-220.

Leeuwenberg, E. L. J. (1971). A perceptual coding language for visual and auditory patterns. American Journal of Psychology, 84, 307-349. 
Leeuwenberg, E. L. J., \& van der Helm, P. A. (2013). Structural information theory: The simplicity of visual form. Cambridge, UK: Cambridge University Press.

Locher, P., \& Wagemans, J. (1993). Effects of element type and spatial grouping on symmetry detection. Perception, 22, 565-587.

Makin, A. D. J., Rampone, G., Pecchinenda, A., \& Bertamini, M. (2013). Electrophysiological responses to visuospatial regularity. Psychophysiology, 50, 1045-1056.

Makin, A. D. J., Wright, D., Rampone, G., Palumbo, L., Guest, M., Sheehan, R., Cleaver, H., \& Bertamini, M. (2016). An electrophysiological index of perceptual goodness. Cerebral Cortex. http://dx.doi.org/10.1093/cercor/bhw255.

Maloney, R. K., Mitchison, G. J., \& Barlow, H. B. (1987). Limit to the detection of glass patterns in the presence of noise. Journal of the Optical Society of America A, 4, 2336-2341.

Marković, S., \& Gvozdenović, V. (2001). Symmetry, complexity and perceptual economy: Effects of minimum and maximum simplicity conditions. Visual Cognition, 8:3-5, 305-327.

Matsuda, T. (1978). Judgments of pattern goodness and intra-configurational transformation structures: An examination of Imai's theory. Japanese Journal of Psychology, 49, 207-214 (Japanese text with English abstract).

Palmer, S. E. (1982). Symmetry, transformation, and the structure of perceptual systems. In J. Beck (Ed.), Organization and representation in perception (pp. 95-144). Hillsdale: Erlbaum.

Palmer, S. E. (1983). The psychology of perceptual organization: A transformational approach. In J. Beck, B. Hope, \& A. Rosenfeld (Eds.), Human and machine vision (pp. 269-339). New York: Academic Press.

Palmer, S. E. (1991). Goodness, Gestalt, groups, and Garner: Local symmetry subgroups as a theory of figural goodness. In G. R. Lockhead, \& J. R. Pomerantz (Eds.), The perception of structure: Essays in honor of Wendell R. Garner (pp. 23-39). Washington, DC: American Psychological Association.
Sekuler, A. B., \& Palmer, S. E. (1992). Perception of partly occluded objects: A microgenetic analysis. Journal of Experimental Psychology: General, 121, 95-111.

Shubnikov, A. V., \& Koptsik, V. A. (1974). Symmetry in science and art. New York: Plenum

Tyler, C. W., \& Hardage, L. (1996). Mirror symmetry detection: Predominance of secondorder pattern processing throughout the visual field. In C. W. Tyler (Ed.), Human symmetry perception and its computational analysis (pp. 157-171).Zeist, The Netherlands: VSP.

van der Helm, P. A. (2010). Weber-Fechner behaviour in symmetry perception? Attention, Perception \& Psychophysics, 72, 1854-1864.

van der Helm, P. A. (2011). The influence of perception on the distribution of multiple symmetries in nature and art. Symmetry, 3, 54-71.

van der Helm, P. A. (2014). Simplicity in vision: A multidisciplinary account of perceptual organization. Cambridge, UK: Cambridge University Press.

van der Helm, P. A., \& Leeuwenberg, E. L. J. (1996). Goodness of visual regularities: A nontransformational approach. Psychological Review, 103, 429-456.

Wagemans, J. (1995). Detection of visual symmetries. Spatial Vision, 9, 9-32.

Wenderoth, P. (1995). The role of pattern outline in bilateral symmetry detection with briefly flashed dot patterns. Spatial Vision, 9, 57-77.

Wenderoth, P. (1996). The effects of the contrast polarity of dot-pair partners on the detection of bilateral symmetry. Perception, 25, 757-771.

Wenderoth, P., \& Welsh, S. (1998). Effects of pattern orientation and number of symmetry axes on the detection of mirror symmetry in dot and solid patterns. Perception, 27. 965-976.

Weyl, H. (1952). Symmetry. Princeton, NJ: Princeton University Press. 